Check for updates

Cite this: J. Mater. Chem. C, 2021, 9, 2801

\section{Non-monotonic magnetic anisotropy behavior as a function of adsorbate coverage in Fe ultrathin films near the spin reorientation transition}

\author{
A. Quesada, (D)*ab G. Chen, ${ }^{\text {a }}$ A. T. N'Diaye, ${ }^{\text {ac }}$ P. Wang, ${ }^{\text {de }}$ Y. Z. Wu ${ }^{\text {de }}$ and \\ A. K. Schmid ${ }^{a}$
}

\begin{abstract}
Carbon monoxide (CO) adsorption on ultrathin fcc Fe films is known to result in the rotation of magnetization from out-of-plane to in-plane. By imaging in real time the magnetic domain structure of perpendicularly magnetized $\mathrm{Fe} /(2 \mathrm{ML}) \mathrm{Ni} / \mathrm{Cu}(100)$ films during exposure to $\mathrm{CO}$, we demonstrate that the effect of adsorption on the magnetic properties occurs in two distinct stages. Initially, when CO bonds preferentially on bridge sites, perpendicular magnetic anisotropy is enhanced. Later, when on-top adsorption dominates, magnetization rotates towards in-plane in agreement with previous studies. This CO-induced spin reorientation transition (SRT) is not reversible by annealing, as CO desorption would require high temperature cycles which yield permanent structural changes in the metal films. This study demonstrates the existence of a novel non-monotonic behavior of the magnetic anisotropy as a function of adsorbate coverage. The influence of $\mathrm{CO}$ on ultrathin film magnetism in real time near critical points, where the anisotropy balance is extremely sensitive to small variations, reveals the previously elusive complexity of this adsorbate induced SRT.
\end{abstract}

Received 4th November 2020, Accepted 26th January 2021

DOI: $10.1039 /$ d0tc05201a

rsc.li/materials-c

\section{Introduction}

The micromagnetic structure of thin magnetic films is determined by a delicate equilibrium between the different contributions to the magnetic free energy. In the ultrathin limit, surface and interface effects often dictate the magnetic properties; however, as thickness increases, magnetocrystalline and magnetostatic terms usually become dominating. At thickness near the transition between interface- and bulk-dominated regimes, this equilibrium can be strongly influenced by subtle morphological, thermal or chemical changes, and many studies of these phenomena have contributed to a deep understanding of the critical parameters that govern magnetic transitions in the ultrathin regime. ${ }^{1,2}$ The detailed understanding of these phenomena permits the control of magnetic properties of thin films to enable applications in technologies such as memory, logic or sensing devices.

In this context, gas adsorption significantly affects the magnetic properties of metallic ultrathin films. ${ }^{1-3}$ For instance, hybridization between metallic atoms and adsorbates results in

\footnotetext{
${ }^{a}$ NCEM, Molecular Foundry, Lawrence Berkeley National Lab, Berkeley, CA 94720, USA.E-mail: a.quesada@icv.csic.es

${ }^{b}$ Instituto de Cerámica y Vidrio, CSIC, 28049, Madrid, Spain

${ }^{c}$ Lawrence Berkeley National Lab, Advanced Light Source, Berkeley, CA 94720, USA

${ }^{d}$ Department of Physics, State Key Laboratory of Surface Physics, Fudan University, Shanghai 200433, China

${ }^{e}$ Shanghai Research Center for Quantum Sciences, Shanghai 201315, China
}

either promotion or inhibition of spin reorientation transitions (SRT) between perpendicular and in-plane magnetization directions. ${ }^{4}$ Hybridization can alter the magnetic moment of the topmost atoms, surface anisotropy can be modified and lattice spacings may vary with magnetoelastic implications. In particular, the adsorbate-induced magnetic properties of $\mathrm{Fe} /$ $\mathrm{Cu}(100)$ ultrathin films have received considerable attention..$^{5-8}$ Hydrogen adsorption promotes a reversible $\mathrm{SRT}^{9}$ and oxygen produces an adsorbate-induced decrease of the critical thickness that favors in-plane magnetization. ${ }^{7}$ In the case of $\mathrm{CO}$ adsorption, it has been reported to reduce the Curie temperature and to promote a SRT towards in-plane in 4 monolayers (ML) Fe films grown both at room temperature (RT) and at $140 \mathrm{~K} \cdot{ }^{5,7,10} \mathrm{Fe} / \mathrm{Cu}(100)$ is a rather complex magnetic system that undergoes several structural and magnetic transitions in overlapping ranges of thickness, including a ferromagnetic/ antiferromagnetic transition at around $4 \mathrm{ML}^{10,11}$ and a SRT at $11 \mathrm{ML}$.

Goal of our study is to bring forward a novel non-monotonic dependence of the magnetic anisotropy of an ultrathin film on adsorbate coverage. The influence of $\mathrm{CO}$ adsorption on the magnetic properties of epitaxial $\mathrm{Fe}(100)$ film is addressed. We investigate the bilayer system $\mathrm{Fe} / \mathrm{Ni} / \mathrm{Cu}(100)$ : this system is similar to the case of fcc $\mathrm{Fe} / \mathrm{Cu}(100)$, while it permits us to avoid the complications associated with antiferromagnetic phase in the $\mathrm{Fe} / \mathrm{Cu}(100)$ system. ${ }^{12,13}$ With the addition of the 
2 ML Ni spacer the system undergoes a direct transition from out-of-plane to in-plane orientation of the magnetization at a thickness of the Fe layer of roughly $3 \mathrm{ML} .{ }^{14}$ In the vicinity of the SRT, an out-of-plane stripe phase forms whose domain width is extremely sensitive to minor changes in the balance of magnetic anisotropies. ${ }^{15}$ This allows us to elucidate the effect of $\mathrm{CO}$ adsorption on ultrathin fcc Fe by imaging in situ the magnetic stripe domain structure of $\mathrm{Fe} / \mathrm{Ni} / \mathrm{Cu}(100)$ using spin-polarized low-energy electron microscopy (SPLEEM).

\section{Experimental}

$\mathrm{A} \mathrm{Cu}(100)$ substrate with miscut angle below $0.1^{\circ}$ was cleaned by cycles of $\mathrm{Ar}^{+}$sputtering and annealing at $600{ }^{\circ} \mathrm{C}$ inside the spin-polarized low-energy electron microscope (SPLEEM) ultrahigh vacuum (UHV) chamber. 2 ML of Ni and 2-3 ML of Fe were deposited by molecular beam epitaxy (MBE) at $293 \mathrm{~K}$ under a base pressure in the range of $10^{-11}$ Torr inside the microscope chamber. The deposition rate, typically of $0.3 \mathrm{ML} \mathrm{min}^{-1}$, was controlled by following LEEM image intensity oscillations associated with layer-by-layer growth.

In a first set of experiments, after growing the Fe layer to the desired thickness-several thicknesses in the 2.5-3 ML range were studied, which corresponds to $0.45-0.54 \mathrm{~nm}-$, CO was dosed through a leak valve into the UHV chamber from a high purity source at $293 \mathrm{~K}$, reaching a pressure of between $1 \times 10^{-10}-3 \times 10^{-10}$ Torr. To determine the dose in Langmuir (L), the pressure reading in Torr was multiplied by a correction factor of 1.05 corresponding to $\mathrm{CO}$ and multiplied by the exposure time. Typically, CO pressures in the low $10^{-10}$ Torr range were employed to allow sufficient time for dose-resolved SPLEEM imaging during exposure. The SPLEEM images were acquired at 1 image per second rate during the exposure to CO.

In a second set, the same experiments were performed at $100 \mathrm{~K}$. The sample was cooled by flowing liquid nitrogen through a capillary inside the microscope chamber, CO exposure and SPLEEM image acquisition was carried out at $100 \mathrm{~K}$ under the same conditions described above. In some cases, and for comparison purposes, the Ni buffer layer was not grown.

In a third set, after exposure to CO both at $293 \mathrm{~K}$ and $100 \mathrm{~K}$, the films were annealed at $400 \mathrm{~K}$ for $10 \mathrm{~min}$, and then cooled back down to $293 \mathrm{~K}$ for image acquisition.

The experiments were performed at the SPLEEM user facility at the National Center for Electron Microscopy at Lawrence Berkeley National Laboratory. ${ }^{16}$ The SPLEEM images that reveal magnetic contrast are obtained as follows. By subtracting pixel-by-pixel two low-energy electron microscopy (LEEM) images recorded with opposite electron polarization vector, pixel intensity in the resulting image represents a map of the magnitude of the component of the magnetization along the spin polarization axis. An electron-optical spin-manipulator allows the spin polarization axis to be adjusted, so that the film magnetization can be mapped along the out-of-plane and any in-plane direction with up to $10 \mathrm{~nm}$ lateral resolution. To maximize contrast, the electron energy was chosen to be $9 \mathrm{eV}$.
Typical image integration time is $1 \mathrm{~s}$, which allows mapping of the magnetic domain structure in real-time. All images presented here were acquired with the spin axis adjusted to image the magnetization component in the direction perpendicular to the surface.

\section{Results and discussion}

The magnetic domain width of the stripe phase as a function of CO dose is presented in Fig. 1 for a perpendicularly magnetized $\mathrm{Fe}(2.4 \mathrm{ML}) / \mathrm{Ni}(2 \mathrm{ML}) / \mathrm{Cu}(100)$ film. Initially, the domain width increases from $0.6 \mu \mathrm{m}$ to $2.3 \mu \mathrm{m}$. At a dose of approximately $0.7 \mathrm{~L}$, the opposite effect is observed: stripe domains become narrower as $\mathrm{CO}$ is adsorbed. At $1 \mathrm{~L}$, magnetization rotates into the in-plane direction resulting in the loss of out-of-plane magnetic contrast. Taking into account that the initial sticking coefficient of $\mathrm{CO}$ on $\mathrm{Fe}$ at $298 \mathrm{~K}$ is close to 1 and previous studies on $\mathrm{CO}$ adsorption on fcc Fe, ${ }^{17,18} 1 \mathrm{~L}$ should correspond to a CO coverage close to saturation. The effect of CO adsorption at RT is thus a two-step process that ultimately results in a CO-induced SRT, in agreement with previously published work. $^{5-7}$ This CO-induced SRT is different from the Fe thickness-induced SRT in $\mathrm{Fe} / \mathrm{Ni} / \mathrm{Cu}(100)$, where the stripe width decreases exponentially as a function of Fe thickness as the SRT is approached. ${ }^{15}$ What's interesting here is that if CO were exclusively promoting in-plane magnetization, then the stripe width should in principle monotonically decrease with CO coverage. Instead, we observe an initial adsorption stage where domain width increases. The existence of plateaus in the blue part of the curve (and not in the red) is probably due to the fact that the energy cost of nucleating/erasing magnetic domains decreases exponentially as the SRT is approached. This implies

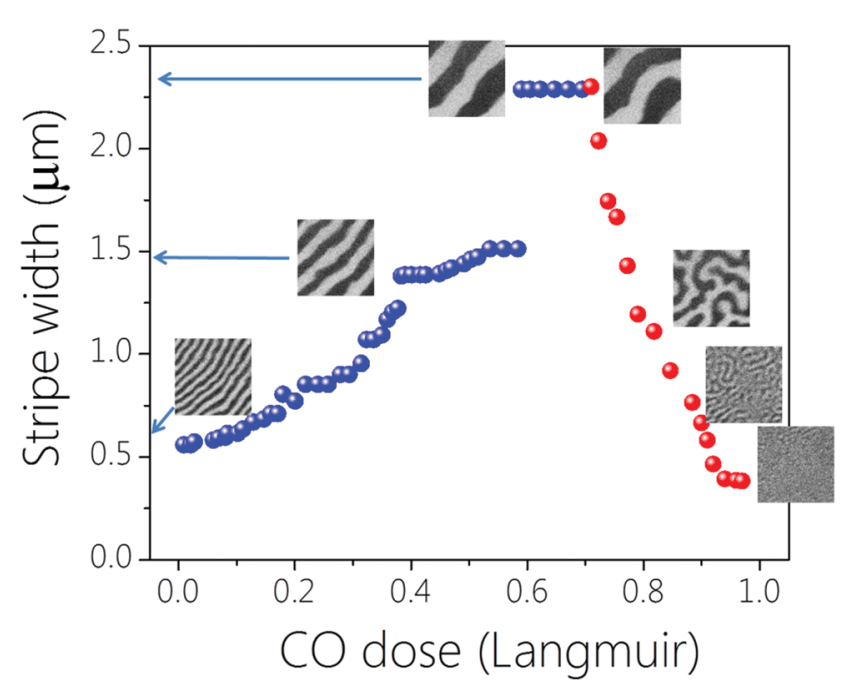

Fig. 1 Magnetic domain width as a function of $\mathrm{CO}$ dose at $293 \mathrm{~K}$ in a perpendicularly magnetized $\mathrm{Fe}(2.4 \mathrm{ML}) \mathrm{Ni}(2 \mathrm{ML}) / \mathrm{Cu}(100)$ film. SPLEEM images with a field-of-view of $13 \mu \mathrm{m}$ showing out-of-plane magnetic contrast at each stage of $\mathrm{CO}$ adsorption are included. Blue arrows indicate width of domains for some images. Blue/red data points correspond to the CO coverages for which perpendicular/in-plane anisotropy are favored respectively. 
the existence of higher energy barriers below $0.7 \mathrm{~L}$ that need to be overcome in order to annihilate domain walls and accommodate wider domains. In addition, local pinning effects, which are stronger for larger domains, may "freeze" a certain domain structure in the limited field of view of the SPLEEM images acquired, thus accentuating the presence of plateaus.

It is worth noting as well that the asymmetry value of the magnetic contrast remained constant up to $0.8 \mathrm{~L}$. A difference in the grey value between black and white stripes of 130 (arbitrary units) is measured below $0.8 \mathrm{~L}$. Above $0.8 \mathrm{~L}$, and close to the SRT, asymmetry decreases. At $0.9 \mathrm{~L}$ for instance, an average grey value difference of 60 is measured, and it tends to zero as the contrast is lost for $>1 \mathrm{~L}$. Given that contrast in SPLEEM is proportional to magnetization, ${ }^{16}$ this suggests that no appreciable changes in the net out-of-plane magnetization of the film are taking place between $0-0.8 \mathrm{~L} \mathrm{CO}$ adsorption. It is likely that the observed decrease in perpendicular asymmetry in the vicinity of the SRT is due, on one hand to the increase in the density of domain walls for a given area (where spins are in-plane), and on the other hand to the spin canting towards in-plane of the spins inside the stripes, which increases as the SRT is approached. ${ }^{14}$

The stripe width is determined by a number of factors, including the magnetic moment at each lattice site and the total effective anisotropy. ${ }^{15}$ In this scenario, these parameters are altered by gas adsorption. In the experiment shown in Fig. 2, 2.8 $\mathrm{ML}$ of $\mathrm{Fe}(0.5 \mathrm{~nm})$ were grown while acquiring SPLEEM images. The image sequence (a)-(f) monitors the out-of-plane magnetization as a function of increasing $\mathrm{Fe}$ thickness. As expected, stripes are seen to become narrower with thickness and magnetic contrast is ultimately lost at the SRT. The panel (f) corresponds to a thickness of $2.8 \mathrm{ML}$ of Fe, which is above the SRT thickness threshold. Beyond this thickness threshold, magnetization lies in-plane, which is the reason for the absence of contrast. Subsequently, after stopping Fe growth, the film was exposed to $\mathrm{CO}$ while continuing to image the out-of-plane magnetization. Images $(\mathrm{g})$ to (l) show how the magnetic stripe phase is progressively recovered at a CO dose slightly below $0.3 \mathrm{~L}$. Further CO dosing resulted in the magnetization rotating back to the in-plane direction (not shown).

The experiment was repeated for several Fe thicknesses and it was found that above $3 \mathrm{ML}$, out-of-plane magnetization could not be restored by $\mathrm{CO}$ adsorption. Thus, besides the changes in magnetic moments that may be occurring, the influence of $\mathrm{CO}$ on the effective anisotropy is unambiguous: at low CO dose perpendicular magnetic anisotropy (PMA) is favored while effective in-plane anisotropy is promoted at high CO dose. Indeed, as explained in previous theoretical models, ${ }^{12}$ a universal dependence of the stripe domain width on the magnetic anisotropy exists which yields wider stripes as the effective PMA increases.

Although CO adsorption may be slightly affecting exchange constants and magnetic moments in the system, ${ }^{8}$ assuming constant exchange values it is possible to estimate the quantitative change in effective anisotropy $K_{\text {eff }}$ as a function of CO exposure. Using the model for the stripe width developed by $\mathrm{Wu}$ et al., ${ }^{12}$ we obtain that right before CO exposure, the effective anisotropy per unit cell of the $2 \mathrm{D}$ lattice is $K_{\mathrm{eff}}=0.08 \mu \mathrm{eV}$. At the maximum stripe width, corresponding to a CO dose of $0.6 \mathrm{~L}, K_{\text {eff }}$ reaches $1.2 \mu \mathrm{eV}$.

To interpret these findings we start by comparing our results with prior work on related systems. In agreement with our observations, two earlier studies reported that CO doses above $1 \mathrm{~L}$ promote an SRT in ultrathin fcc $\mathrm{Fe} / \mathrm{Cu}(100)$ at $100 \mathrm{~K} .{ }^{5,7} \mathrm{In}$ order to compare with these studies, we investigate the effect of $\mathrm{CO}$ adsorption both at RT and $100 \mathrm{~K}$, with and without $\mathrm{Ni}$ spacer layer, in Fe films that presented out-of-plane magnetization. Table 1 summarizes the results. For $\mathrm{Fe}(4 \mathrm{ML}) / \mathrm{Cu}(100)$ films, $\mathrm{CO}$ adsorption at $100 \mathrm{~K}$ reproduces the results reported by Abe et al.: ${ }^{5}$ magnetization direction monotonically tends to in-plane upon CO exposure. At RT, after $1 \mathrm{~L}$ CO adsorption, magnetic contrast is lost, both in the in-plane and out-of-plane directions. For $\mathrm{Fe}(2.4 \mathrm{ML}) / \mathrm{Ni}(2 \mathrm{ML}) / \mathrm{Cu}(100)$ films, at $100 \mathrm{~K}$ the

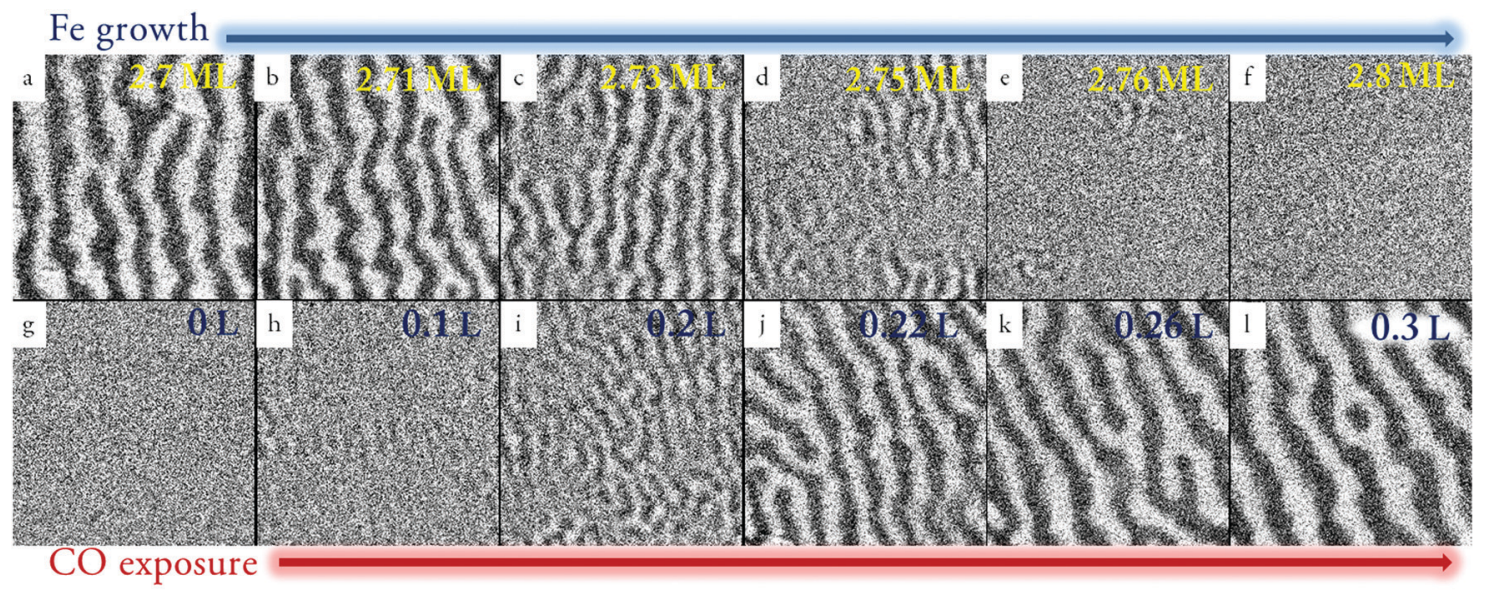

Fig. 2 (a)-(f) Sequence of SPLEEM images corresponding to the growth of Fe on Ni(2 ML)/Cu(100). Initial thickness in (a) is $2.7 \mathrm{ML}$ and final thickness in (f) is $2.8 \mathrm{ML}$. (g)-(l) Sequence of SPLEEM images corresponding to the subsequent exposure to CO of the $2.8 \mathrm{ML}$ Fe film. Electron beam polarization is sensitive to perpendicular magnetization in all cases. 
Table 1 Summary of the effect of CO adsorption on the magnetic properties at $100 \mathrm{~K}$ and $293 \mathrm{~K}$ for the two systems studied

\begin{tabular}{lll}
\hline Exposure temperature & $100 \mathrm{~K}$ & $293 \mathrm{~K}$ \\
\hline$(4 \mathrm{ML}) \mathrm{Fe} / \mathrm{Cu}(100)$ & In-plane anisotropy favored (SRT at $4 \mathrm{~L})$ & No magnetic contrast at $1 \mathrm{~L}$ \\
$\mathrm{Fe} / \mathrm{Ni} / \mathrm{Cu}(100)$ & In-plane anisotropy favored (SRT at $1 \mathrm{~L})$ & PMA favored below $0.7 \mathrm{~L}$ \\
& & In-plane anisotropy favored above $0.7 \mathrm{~L}$ (SRT at $1 \mathrm{~L}$ )
\end{tabular}

effect of CO is again monotonic, promoting the SRT towards in-plane. Therefore, PMA is enhanced only when $\mathrm{Fe} / \mathrm{Ni} / \mathrm{Cu}$ films are exposed to CO at RT.

In addition, the reversibility of the process upon CO desorption stimulated by annealing was investigated. An in-plane magnetized $\mathrm{Fe}(2.7 \mathrm{ML}) / \mathrm{Ni}(2 \mathrm{ML}) / \mathrm{Cu}(100)$ film was exposed to $0.5 \mathrm{~L}$ of $\mathrm{CO}$. The stripe phase was recovered as Fig. 3(a) shows. The film was then annealed at $400 \mathrm{~K}$ for $10 \mathrm{mins}$ in order to trigger CO desorption. Fig. 3(b) shows a SPLEEM image obtained at RT, after the $400 \mathrm{~K}$ annealing, where out-of-plane contrast is detected. Thus, the inplane magnetization of the system prior to $\mathrm{CO}$ adsorption is not recovered after annealing, even if CO is presumably fully desorbed at $400 \mathrm{~K}$, since top Co configurations desorb at $313 \mathrm{~K}$ while bridge sites desorb at $380 \mathrm{~K} \cdot{ }^{19}$ We assume that temperature induced structural changes, such as the appearance of dislocations and local fcc-bcc transitions, or interdiffusion are the plausible causes for this observation. ${ }^{20}$ In fact, an irreversible inverse SRT was also reported in $\mathrm{Fe} / \mathrm{Cu}(100)$ annealed at $350 \mathrm{~K},{ }^{7}$ that was ascribed to irreversible structural changes. These structural changes may be the reason for the wider stripes observed after annealing as well.

The observed two stages of micromagnetic effects of $\mathrm{CO}$ adsorption may be attributed to the CO adsorption process, which takes place in two stages. ${ }^{19,21}$ Initially, and up to $0.6 \mathrm{~L}$, $\mathrm{CO}$ adsorbs on fcc $\mathrm{Fe}(2 \mathrm{ML}) / \mathrm{Cu}(100)$ preferentially on Fe bridge sites. In a second step, which starts at exposures around $0.4 \mathrm{~L}$, CO adsorbs molecularly at on-top locations. Above $1 \mathrm{~L}$, nearly all CO molecules are adsorbed in on-top bonding conditions. The fact that CO adsorption and its effect on magnetic properties both present a two-step behavior, and that the CO exposures associated with the initial and the final regime perfectly

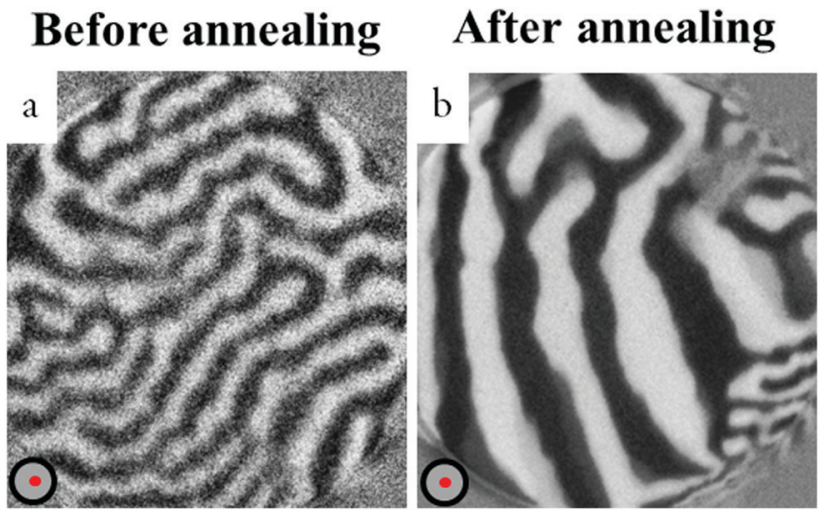

Fig. 3 (a) SPLEEM image at $293 \mathrm{~K}$ showing out-of-plane magnetic contrast of the $\mathrm{Fe}(2.7 \mathrm{ML}) / \mathrm{Ni}(2 \mathrm{ML}) / \mathrm{Cu}(100)$ film after exposure to $5 \mathrm{~L}$ of CO. (b) SPLEEM image of the same film after annealing at $400 \mathrm{~K}$ for $10 \mathrm{~min}$ to promote $\mathrm{CO}$ desorption. Red dots indicate that magnetization is out-of-plane in both images. coincide, points to the interpretation that $\mathrm{CO}$ adsorption on bridge sites promotes PMA while on-top adsorption favors in-plane effective anisotropy. Given that both bridge and on-top sites are stable at $100 \mathrm{~K}$ and $293 \mathrm{~K}$, we suggest that the temperature dependence of the relevant magnetic quantities - magnetic moment, surface, shape and magnetocrystalline anisotropy - is the reason for the different behavior at the two temperatures studied. It is important to point out in any case that the influence of preferred adsorption at atomic steps/terraces for low/high CO doses respectively cannot be discarded as a contributing factor to our observations.

The PMA enhancement could be the consequence of different physical effects. The magnetic anisotropy energy (MAE) per unit volume is defined as:

$$
\mathrm{MAE}=-(1 / 2) \mu_{0} M_{\mathrm{S}}^{2}+\left(K_{\mathrm{i}}+K_{\mathrm{S}}\right) / d+K_{\mathrm{V}}
$$

where $M_{\mathrm{S}}$ is the saturation magnetization, $K_{\mathrm{i}}, K_{\mathrm{S}}$ and $K_{\mathrm{V}}$ are interface, surface and volume contributions to the second order anisotropy respectively and $d$ is the film thickness. Previous works have theoretically and experimentally determined that, depending on the fcc Fe film thickness, a substantial reduction in the magnetic moment of the Fe atoms at the surface can take place during $\mathrm{CO}$ adsorption. ${ }^{5,7,8}$ In fact, this magnetic moment decrease has been postulated as the main reason for the CO-induced SRT from out-of-plane to in-plane reported by Abe et al. ${ }^{5}$ However, the absence of magnetic contrast variations in SPLEEM images below $0.8 \mathrm{~L}$ indicates that total magnetization remains approximately constant in our system. This suggests that the main contribution to PMA of CO bridge bonding is the increase of surface anisotropy. Orbital hybridization between $\mathrm{C}$ p orbitals and transition metal d orbitals has been reported to suppress negative contributions to $K_{\mathrm{S}}$, thus promoting PMA. $^{22,23}$ We suggest a similar effect is occurring in our system. On the contrary, top CO bonding must be enhancing positive contributions to $K_{\mathrm{S}}$. The electron density and charge transfer between Fe d orbitals and Co $\pi$ orbitals strongly depend on bond geometry. ${ }^{8}$ Given that different orbitals are involved for bridge and top bonding, it seems plausible that the local hybridization differences are responsible for the different effect on the MAE of each adsorption site. $A b$ initio calculations are needed to further elucidate and quantify the effect.

CO-induced PMA is only observed under very specific conditions near the stripe phase SRT and at RT which implies that the effect is weak and highlights the sensitivity of the stripe phase to subtle changes in anisotropy constants. In this sense, our results highlight the suitability of SPLEEM for this type of experiments. ${ }^{24}$ The ability of SPLEEM to provide vector magnetization contrast at a rate of 1 image per $s$ in UHV conditions and during both MBE growth and exposure to adsorbates cannot be matched by any other magnetic characterization 
technique, which normally employ times longer than 1 minute to deliver an image.

\section{Conclusions}

In summary, we have observed a CO-induced two-step effect on the magnetic properties of $\mathrm{Fe} / \mathrm{Ni} / \mathrm{Cu}(100)$ films. At low coverages, PMA is enhanced and CO can even trigger an inverse SRT from in-plane to out-of-plane magnetization. As CO adsorption increases, the opposite effect is observed and an SRT towards in-plane is favored in initially perpendicularly magnetized films. Upon CO desorption by annealing, irreversible structural changes occur that preclude the reversibility of the effect. Selective molecular adsorption at bridge sites (for low coverages) or on-top sites (high coverages) is suggested as causing the distinct response of the effective anisotropy as a function of CO coverage. Our findings underline the suitability of studies near critical points for unraveling the influence of adsorbates in the magnetic properties of ultrathin films, by revealing a surprising non-monotonic dependence of the anisotropy on CO coverage.

\section{Conflicts of interest}

There are no conflicts to declare.

\section{Acknowledgements}

The experiments, performed at the Molecular Foundry, National Center for Electron Microscopy, Lawrence Berkeley National Laboratory, were supported by the Office of Science, Office of Basic Energy Sciences, Scientific User Facilities Division, of the U.S. Department of Energy under Contract No. DE-AC02-05CH11231. A. Q. acknowledges financial support from the Spanish Ministerio de Ciencia e Innovación through Proj. No. RTI2018-095303-A-C52 and the "Ramón y Cajal" Program (RYC-2017-23320); and from the European Commission through project AMPHIBIAN H2020-NMBP-2016AMPHIBIAN (no. 720853). Y. Z. W. acknowledges financial support by National Natural Science Foundation of China (Grants No. 11734006 and No. 11974079), and Shanghai Municipal Science and Technology Major Project (Grant No. 2019SHZDZX01).

\section{References}

1 P. D. Johnson, Rep. Prog. Phys., 1997, 60, 1217.

2 C. F. Vaz, J. C. Bland and G. Lauhoff, Rep. Prog. Phys., 2008, 71, 056501.

3 C. R. Abeledo and P. W. Selwood, J. Chem. Phys., 1962, 37, 2709.
4 B. Santos, S. Gallego, A. Mascaraque, K. F. McCarty, A. Quesada, A. T. N'Diaye, A. K. Schmid and J. De La Figuera, Phys. Rev. B: Condens. Matter Mater. Phys., 2012, 85, 134409.

5 H. Abe, K. Amemiya, D. Matsumura, J. Miyawaki, E. Sako, T. Ohtsuki, E. Sakai and T. Ohta, Phys. Rev. B: Condens. Matter Mater. Phys., 2008, 77, 054409.

6 H. Abe, K. Amemiya, J. Miyawaki, E. O. Sako, M. Sakamaki, D. Matsumura, T. Ohtsuki, E. Sakai and T. Ohta, e-J. Surf. Sci. Nanotechnol., 2008, 6, 233.

7 D. Peterka, A. Enders, G. Haas and K. Kern, Phys. Rev. B: Condens. Matter Mater. Phys., 2002, 66, 104411.

8 D. Spišák and J. Hafner, Phys. Rev. B: Condens. Matter Mater. Phys., 2001, 64, 094418.

9 R. Vollmer and J. Kirschner, Phys. Rev. B: Condens. Matter Mater. Phys., 2000, 61, 4146.

10 J. Thomassen, F. May, B. Feldmann, M. Wuttig and H. Ibach, Phys. Rev. Lett., 1992, 69, 3831.

11 K. Kalki, D. D. Chambliss, K. E. Johnson, R. J. Wilson and S. Chiang, Phys. Rev. B: Condens. Matter Mater. Phys., 1993, 48, 18344.

12 Y. Z. Wu, C. Won, A. Scholl, A. Doran, H. W. Zhao, X. F. Jin and Z. Q. Qiu, Phys. Rev. Lett., 2004, 93, 117205.

13 J. Choi, J. Wu, C. Won, Y. Z. Wu, A. Scholl, A. Doran, T. Owens and Z. Q. Qiu, Phys. Rev. Lett., 2007, 98, 207205.

14 G. Chen, J. Zhu, A. Quesada, J. Li, A. T. N'Diaye, Y. Huo, T. P. Ma, Y. Chen, H. Y. Kwon, C. Won, Z. Q. Qiu, A. K. Schmid and Y. Z. Wu, Phys. Rev. Lett., 2013, 110, 177204.

15 C. Won, Y. Z. Wu, J. Choi, W. Kim, A. Scholl, A. Doran, T. Owens, J. Wu, X. F. Jin, H. W. Zhao and Z. Q. Qiu, Phys. Rev. B: Condens. Matter Mater. Phys., 2005, 71, 224429.

16 N. Rougemaille and A. K. Schmid, Eur. Phys. J.: Appl. Phys., 2010, 50, 20101.

17 T. Wadayama, K. Kubo, T. Yamashita, T. Tanabe and A. Hatta, Appl. Surf. Sci., 2002, 199, 254.

18 O. L. J. Gijzeman, T. J. Vink, O. P. van Pruissen and J. W. Geus, J. Vac. Sci. Technol., A, 1987, 5, 718.

19 T. Tanabe, Y. Suzuki, T. Wadayama and A. Hatta, Surf. Sci., 1999, 427-428, 414.

20 E. Mentz, D. Weiss, J. E. Ortega, A. Bauer and G. Kaindl, J. Appl. Phys., 1997, 82, 482.

21 T. Tanabe, T. Shibahara, R. Buckmaster, T. Ishibashi, T. Wadayama and A. Hatta, Surf. Sci., 2000, 466, 1.

22 K. Bairagi, A. Bellec, V. Repain, C. Fourmental, C. Chacon, Y. Girard, J. Lagoute, S. Rousset, L. Le Laurent, A. Smogunov and C. Barreteau, Phys. Rev. B, 2018, 98, 1.

23 D. Li, C. Barreteau and A. Smogunov, Phys. Rev. B, 2016, 93, 1.

24 G. Chen, A. Mascaraque, H. Jia, B. Zimmermann, M. Robertson, R. Lo Conte, M. Hoffmann, M. A. González Barrio, H. Ding, R. Wiesendanger, E. G. Michel, S. Blügel, A. K. Schmid and K. Liu, Sci. Adv., 2020, 6, eaba4924. 